\title{
Editorial: Structure, Function, and Evolution of E3 Ligases and Targets
}

\author{
Derek J. Gingerich ${ }^{1 *}$, Hanjo Hellmann ${ }^{2}$, Matthew J. Christians ${ }^{3}$ and Sophia L. Stone ${ }^{4}$ \\ ${ }^{1}$ Department of Biology, University of Wisconsin-Eau Claire, Eau Claire, WI, United States, ${ }^{2}$ School of Biological Sciences, \\ Washington State University, Pullman, WA, United States, ${ }^{3}$ Department of Cell and Molecular Biology, Grand Valley State \\ University, Allendale, MI, United States, ${ }^{4}$ Department of Biology, Dalhousie University, Halifax, NS, Canada
}

Keywords: ubiquitin (Ub), E3 Ub-ligase, ubiquitin-like proteins (UBLs), ubiquitylation (Ubiquitination), ubiquitin 26S-proteasome system, E3-ligases

\section{Editorial on the Research Topic}

Structure, Function, and Evolution of E3 Ligases and Targets

\section{INTRODUCTION}

To respond to the environment and regulate growth and development, it is critical for organisms to precisely modulate the activity of proteins in their cells. One mechanism by which this regulation occurs is the covalent attachment of ubiquitin (Ub) and other ubiquitin-like proteins (UBLs) to specific target proteins. These modifications can alter the function, location, or levels of the target. Attachment of the majority of these modifiers occurs by an ATP-dependent E1/E2/E3 conjugation cascade during which a thioester linkage is formed between the Ub/UBL and the E1, followed by a transthiolation reaction where the $\mathrm{Ub} / \mathrm{UBL}$ is transferred to the $\mathrm{E} 2$, then attachment of the $\mathrm{Ub} / \mathrm{UBL}$ to the target protein via an isopeptide bond. This final step is typically mediated by $\mathrm{E} 3$ ligases, which recruit the E2, bind the substrate, and catalyze linkage between the Ub/UBL and the target.

\section{OPEN ACCESS}

Edited and reviewed by: Els JM Van Damme,

Ghent University, Belgium

*Correspondence: Derek J. Gingerich gingerdj@uwec.edu

Specialty section: This article was submitted to Plant Physiology, a section of the journal Frontiers in Plant Science

Received: 30 August 2021 Accepted: 13 September 2021 Published: 11 October 2021

Citation:

Gingerich DJ, Hellmann H, Christians MJ and Stone SL (2021)

Editorial: Structure, Function, and Evolution of E3 Ligases and Targets.

Front. Plant Sci. 12:767281.

doi: 10.3389/fpls.2021.767281

\section{UBL E3-LIGASES}

UBLs which use an E1/E2/E3-like reaction cascade for target attachment include SUMO (Small Ublike Modifier), RUB (Related to Ubiquitin, called Nedd8 in yeast and animals), ATG8 (AutophagyRelated 8) and ATG12 (which are conjugated to the lipid phosphatidylethanolamine and the ATG5 protein, respectively), and UFM1 (Ubiquitin-Fold Modifier 1) (Vierstra, 2012; Callis, 2014; Daniel and Liebau, 2014; Augustine and Vierstra, 2018; Witting and Mulder, 2021). The attachment of UBLs to targets is mediated by small families of E3-ligases (Figure 1). An example is SUMO and in this Research Topic Jmii and Cappadocia describe this interesting group of E3s involved in SUMOylation, which serves as a regulatory tool to change subcellular localization, complex function, or the protein-protein interactions of targets. Although plants encode very few SUMO E3-ligases, they broadly affect abiotic stress and hormonal responses, developmental processes, and cell-cycle regulation. Further insights into the structural organization and details on cellular processes are provided in the review (Jmii and Cappadocia).

\section{UB E3-LIGASES}

In contrast to the E3s involved in UBL conjugation, Ub-E3 ligases are a huge and diverse group. They divide into three main types: the RING (Really-Interesting New Gene) family, the HECT (Homologous to the E6AP Carboxyl Terminus) family, and the RBR (RING-in-Between-RING) family (Downes et al., 2003; Hua and Vierstra, 2011; Callis, 2014; Dove and Klevit, 2017). The 
A

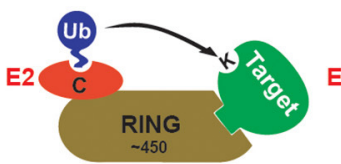

RING

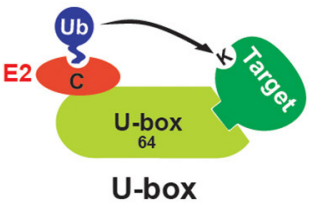

U-box

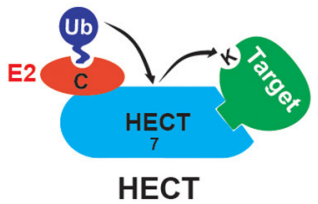

HECT

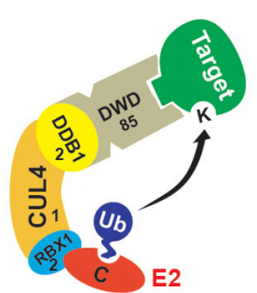

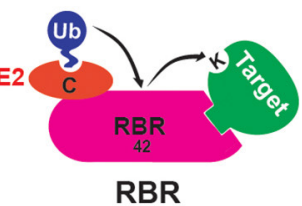

RBR

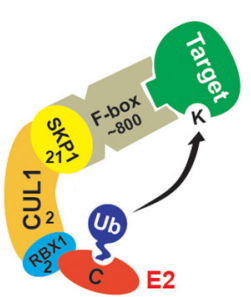

SCF CRL

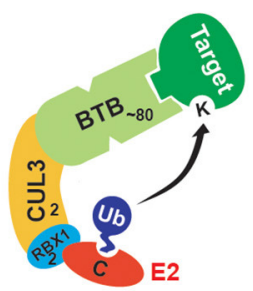

BTB/CUL3 CRL

DDB1/DWD/CUL4 CRL

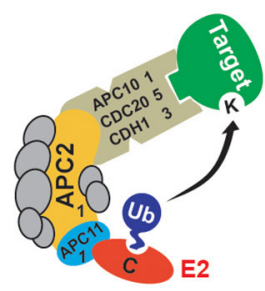

$\mathrm{APC} / \mathrm{C}$

B
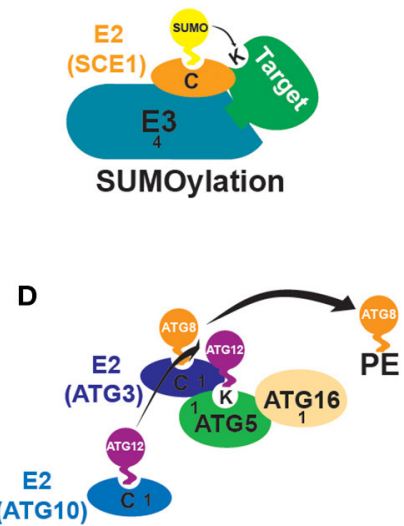

ATG8/12 Conjugation

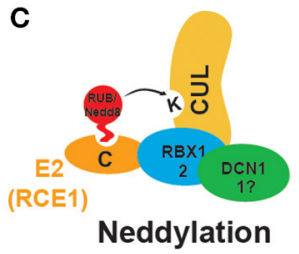

E

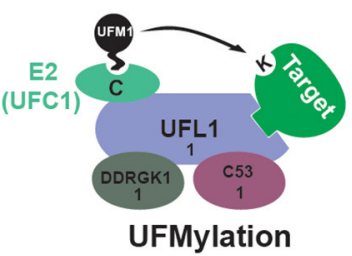

FIGURE 1 | Subunit composition of E3-ligases in plants. The numbers displayed within each subunit indicate the number of genes in the Arabidopsis thaliana genome putatively encoding the E3 ligase or E3 ligase component. (A) Schematics of E3 ubiquitin (Ub)-ligases in plants. RING (Really-Interesting New Gene) and U-box E3 ligases bind an E2 and the target and facilitate direct transfer of Ub to the target protein. HECT (Homologous to the E6AP Carboxyl Terminus) and RBR (RING-in-Between-RING) E3 ligases transfer the Ub from the E2 to a cysteine residue in the E3, then from the cysteine to the target protein. The CRLs (Cullin-RING Ligases) are multi-subunit structures with a Cullin (CUL) backbone protein that binds the E2 and a target adapter protein/complex. In SCF (Skp1/CUL1/F-box) complexes target adaptor F-box proteins bind to an Skp1 (S-phase Kinase-associated Protein 1)-like protein which interacts with CUL1. In the CRL3 complexes, a BTB (Broad-Complex, Tramtrack, and Bric-à-Brac) domain-containing protein binds to the target and CUL3. In the CRL4 complexes, WD40 domain-containing DWD (DDB1 Binding WD40) proteins bind the targets and interact with CUL4 through a DDB1 (Damaged DNA Binding 1) protein bridge. The APC/C (Anaphase-Promoting Complex/Cyclosome) contains at least 11 subunits. APC2 is a Cullin-like protein and APC11 is similar to RBX1 (Ring Box Protein 1). Target recognition occurs via the CDC20 (Cell Division Cycle 20), CDH1 (CDC20 Homolog 1), and APC10 subunits (Gray et al., 2002; Lechner et al., 2002; Schroeder et al., 2002; Capron et al., 2003; Downes et al., 2003; Gingerich et al., 2005; Stone et al., 2005; Kong et al., 2007; Lee et al., 2008; Lima et al., 2010; Marín, 2010; Trujillo, 2018; Saleme et al., 2021). (B) Schematic of the SUMO (Small Ub-like Modifier) E3-ligases. While SUMO E2s can directly interact with target proteins, the E3-ligases stimulate SUMO discharge by the E2 (Augustine and Vierstra, 2018) (https://www.frontiersin.org/articles/10.3389/fpls.2021.652170/full). (C) Proteins involved in the neddylation of Cullins. RBX1 functions synergistically with DCN1 (Defective in Cullin Neddylation 1) to facilitate the transfer of RUB (Related to Ubiquitin)/Nedd8 from the E2 RCE1

(RUB-Conjugating Enzyme 1) to Cullins. There are three possible homologs of DCN1 in Arabidopsis, though two lack the UBA (Ubiquitin-Associated) domain seen in other DCN1 proteins (Merlet et al., 2009; Hosp et al., 2014). (D) Schematic of the ATG8 (Autophagy-Related 8)/ATG12 conjugation pathway. The UBL ATG12 forms a thioester linkage with the E2 enzyme ATG10 and then is conjugated to ATG5. The UBL ATG8 is transferred to the E2 enzyme ATG3, then the ATG5-ATG12 conjugate, together with ATG16, acts as an E3 facilitating ATG8-phosphatidylethanolamine (PE) conjugation (Doelling et al., 2002; Bassham et al., 2006; Mizushima, 2020). (E) UFM1 (Ubiquitin-Fold Modifier 1) conjugation. UFL1 (UFM1-Specific Ligase 1) is the E3-ligase for UFM1 and catalyzes the UFMylation of multiple substrates. UFL1 ligase activity requires interaction with an adaptor protein called DDRGK1 (DDRGK Domain-Containing Protein 1). Another UFL1 interactor, CDK5RAP3 (CDK5 Regulatory Subunit-Associated Protein 3), affects the UFMylation profile and may serve as a substrate adaptor. DDRGK1 and CDK5RAP3 homologs are present in Arabidopsis; the CDK5RAP3 homolog is called C53 (Daniel and Liebau, 2014; Stephani et al., 2021; Witting and Mulder, 2021). 
RING family divides into multiple subfamilies, including monomeric, dimeric, and heteromeric RINGs, the U-box type, the APC/C (Anaphase-Promoting Complex/Cyclosome), and the Cullin-RING ligases (CRLs) (Figure 1) (Stone et al., 2005; Vierstra, 2009; Chen and Hellmann, 2013). Higher eukaryotes encode large numbers of Ub-E3 ligases in their genomes, and this is particularly so in plants (Grau-Bové et al., 2015). Arabidopsis thaliana, for instance, has $\sim 1,500$ loci encoding potential Ub E3s or E3 subunits (Figure 1). Many of these encode the subunits involved in target recognition. For instance, in Arabidopsis, there are $\sim 800$ genes encoding F-box proteins, the target adaptors in the SCF (Skp1/CUL1/F-box) CRL complexes (Hua et al., 2011; Hua, 2021), 450 genes encoding the singlesubunit RING E3s (Stone et al., 2005), and $\sim 80$ genes encoding the BTB (Broad-Complex, Tramtrack, and Bric-à-Brac) protein target adaptors of the BTB/Cullin $3 \mathrm{CRL}$ complexes (Gingerich et al., 2005) (Figure 1). Besides being large, plant E3 families have also undergone dynamic evolution, with diversification in family composition and sizes, and a high rate of gene gains and losses (Grau-Bové et al., 2015; Hua and Yu, 2019; Hua, 2021). This polymorphic nature complicates our ability to predict which family members are active or occur as pseudogenes and present challenges to researchers hoping to elucidate their roles. For these reasons, to date, the functions and targets of the majority of E3 substrate adaptors have not been determined. For instance, among the members of the relatively well-characterized Arabidopsis family of BTB proteins putative biological functions have been identified for only 39 (Christie et al., 2018; Ban and Estelle, 2021). For the F-box family, the percentage is even lower, with just 83 F-box protein-encoding genes $(\sim 10 \%)$ having a function identified via genetic characterization (Hua, 2021). With the majority uncharacterized, determining which are worth spending resources to investigate can be difficult. The development of better approaches to predict and separate active genes from pseudogenes would be crucial. In this Research Topic, Li et al. present such a method, using the F-box gene superfamily in Arabidopsis to demonstrate a novel neural network machine learning approach to classify genes as either functionally active or inactive. By integrating numerous features, their approach may be used to identify active gene candidates, allowing future work to focus on family members most likely to play roles in plant growth and development (Li et al.).

Ultimately, a full understanding of any individual E3-ligase requires dedicated study using a variety of approaches and here we present several articles that describe the characterization of individual E3s and/or their targets. Linden et al. used genetics to characterize the Arabidopsis thaliana RING E3 BRIZ, a heteromeric ligase that contains the BRIZ1 (BRAP2RING-Znf Domain 1) and BRIZ2 proteins. They show that briz1 and briz2 mutants fail to germinate or arrest early in seedling development and that phenotype is reduced when abscisic acid (ABA) levels are reduced. While the target(s) of BRIZ are still unknown, their data suggest that the function of the BRIZ ligase is to suppress ABA response early in development (Linden et al.) The research article from Beathard et al. focuses on a three members of the S23 subfamily of MYB transcription factors. These targets of the ubiquitin proteasome pathway are likely regulated by CUL3-dependent CRLs that use the BTB BPM (BTB/POZ-MATH) proteins as substrate adaptors. Genetic approaches, including overexpression and down-regulation via a polycistronic microRNA, implicate this MYB subfamily in stress and ABA response pathways, adding another facet to abiotic stress-regulation in context with the ubiquitin proteasome pathway (Beathard et al.). The RING E3 ligase COP1 (Constitutive Photomorphogenesis 1) is a longknown master regulator controlling the switch from skototo photomorphogenesis in plants. The review article from Ponnu and Hoecker describes the regulatory steps that control COP1 activities, and nicely outlines this highly complex topic with insights into how this E3 ligase integrates different light qualities in concert with other proteins. In addition to discussing its role in other processes, such as temperature response, hormonal signaling, or cell division, a comparison between COP1 in plants and animals is provided (Ponnu and Hoecker). Finally, Erffelinck et al. describe the use of a yeast twohybrid protein-protein interaction screen to identify the binding partners of the Medicago truncatula RING membrane-anchortype E3 MKB1 (MAKIBISHI1). MKB1 recruits the ER-associated degradation (ERAD) machinery to regulate levels of the enzyme HMGR (3-Hydroxy-3-Methylglutaryl-CoA Reductase), which is a rate-limiting enzyme in the mevalonate pathway that supplies building blocks for the production of triterpene defense compounds. The researchers were able to identify an E2 enzyme for MKB1 and a heat shock protein that interacts with MKB1 and supports its activity. This work thus identifies additional key components of the MKB1-dependent ERAD machinery (Erffelinck et al.).

\section{TRANSLATING E3-LIGASE RESEARCH TO THE FIELD}

The articles in this Research Topic illustrate the wide range of developmental and environmental-response pathways in which E3-ligases are involved. Among these are pathways of agronomic significance, such as those that respond to abiotic stresses including drought, salinity, and temperature. Melo et al. provide a review of what is known about the roles of E3-ligases in abiotic stress responses in rice, with a focus on the monomeric RING and U-box E3s. In addition to providing a detailed description of individual E3s, they also summarize key areas for which more investigation is needed to better understand how these E3s function in these responses. Finally, they suggest stress response-related E3s could be targeted for genetic manipulation, allowing for the production of crops better suited for stress-prone environments (Melo et al.).

\section{CONCLUSIONS AND FUTURE DIRECTIONS}

In summary, this Research Topic presents significant contributions to understanding of E3-ligases. However, there is still much to be done to fully elucidate their functions. 
The targets of the majority of E3 ligases and the pathways in which they operate have yet to be determined. How E3/target interactions are regulated, the structures of the E3/target complexes, and how the E3s and the targets have co-evolved are all areas about which much more still needs to be learned. Increased understanding of these ligases may lead to new E3-centered strategies to modulate responses to biotic and abiotic stresses to improve crop agronomic performance. We anticipate that the study of E3-ligases and the $\mathrm{Ub} / \mathrm{Ubl}$ conjugation pathways will continue to be a highly active, fruitful

\section{REFERENCES}

Augustine, R. C., and Vierstra, R. D. (2018). SUMOylation: re-wiring the plant nucleus during stress and development. Curr. Opin. Plant Biol. 45, 143-154. doi: 10.1016/j.pbi.2018.06.006

Ban, Z., and Estelle, M. (2021). CUL3 E3 ligases in plant development and environmental response. Nat. Plants. 7, 6-16. doi: 10.1038/s41477-020-00833-6

Bassham, D. C., Laporte, M., Marty, F., Moriyasu, Y., Ohsumi, Y., Olsen, L. J., et al. (2006). Autophagy in development and stress responses of plants. Autophagy 2, 2-11. doi: 10.4161/auto.2092

Callis, J. (2014). The ubiquitination machinery of the ubiquitin system. Arabidopsis Book 12:e0174. doi: 10.1199/tab.0174

Capron, A., Okrész, L., and Genschik, P. (2003). First glance at the plant APC/C, a highly conserved ubiquitin-protein ligase. Trends Plant Sci. 8, 83-89. doi: 10.1016/S1360-1385(02)00028-6

Chen, L., and Hellmann, H. (2013). Plant E3 ligases: flexible enzymes in a sessile world. Mol. Plant. 6, 1388-1404. doi: $10.1093 / \mathrm{mp} / \mathrm{sst} 005$

Christie, J. M., Suetsugu, N., Sullivan, S., and Wada, M. (2018). Shining light on the function of NPH3/RPT2-Like proteins in phototropin signaling. Plant Physiol. 176, 1015-1024. doi: 10.1104/pp.17.00835

Daniel, J., and Liebau, E. (2014). The ufm1 cascade. Cells 3, 627-638. doi: $10.3390 /$ cells3020627

Doelling, J. H., Walker, J. M., Friedman, E. M., Thompson, A. R., and Vierstra, R. D. (2002). The APG8/12-activating enzyme APG7 is required for proper nutrient recycling and senescence in Arabidopsis thaliana. J. Biol. Chem. 277, 33105-33114. doi: 10.1074/jbc.M204630200

Dove, K. K., and Klevit, R. E. (2017). RING-Between-RING E3 ligases: emerging themes amid the variations. J. Mol. Biol. 429, 3363-3375. doi: 10.1016/j.jmb.2017.08.008

Downes, B. P., Stupar, R. M., Gingerich, D. J., and Vierstra, R. D. (2003). The HECT ubiquitin-protein ligase (UPL) family in Arabidopsis: UPL3 has a specific role in trichome development. Plant J. 35, 729-742. doi: 10.1046/j.1365-313X.2003.01844.x

Gingerich, D. J., Gagne, J. M., Salter, D. W., Hellmann, H., Estelle, M., $\mathrm{Ma}$, L., et al. (2005). Cullins $3 \mathrm{a}$ and $3 \mathrm{~b}$ assemble with members of the broad complex/tramtrack/bric-a-brac (BTB) protein family to form essential ubiquitin-protein ligases (E3s) in Arabidopsis. J. Biol. Chem 280, 18810-18821. doi: 10.1074/jbc.M413247200

Grau-Bové, X., Sebé-Pedrós, A., and Ruiz-Trillo, I. (2015). The eukaryotic ancestor had a complex ubiquitin signaling system of archaeal origin. Mol. Biol. Evol. 32, 726-739. doi: 10.1093/molbev/msu334

Gray, W. M., Hellmann, H., Dharmasiri, S., and Estelle, M. (2002). Role of the Arabidopsis RING-H2 protein RBX1 in RUB modification and SCF function. Plant Cell 14, 2137-2144. doi: 10.1105/tpc.003178

Hosp, J., Ribarits, A., Retzer, K., Jin, Y., Tashpulatov, A., Resch, T., et al. (2014). A tobacco homolog of DCN1 is involved in pollen development and embryogenesis. Plant Cell Rep. 33, 1187-1202. doi: 10.1007/s00299-0141609-4

Hua, Z. (2021). Diverse evolution in 111 plant genomes reveals purifying and dosage balancing selection models for F-box genes. Int. J. Mol. Sci. 22:871. doi: 10.3390/ijms22020871 field, and we encourage more to join us in the study of these fascinating regulators.

\section{AUTHOR CONTRIBUTIONS}

DG was the primary author of this editorial. $\mathrm{HH}$ and MC provided summaries of articles that are used in the editorial and provided critical review of the editorial. SS provided critical review of the editorial. All four individuals were editors for the research topic.

Hua, Z., and Vierstra, R. D. (2011). The cullin-RING ubiquitinprotein ligases. Annu. Rev. Plant Biol. 62, 299-334. doi: 10.1146/annurev-arplant-042809-112256

Hua, Z., and Yu, P. (2019). Diversifying evolution of the Ubiquitin-26S proteasome system in Brassicaceae and Poaceae. Int. J. Mol. Sci. 20:3226. doi: 10.3390/ijms20133226

Hua, Z., Zou, C., Shiu, S. H., and Vierstra, R. D. (2011). Phylogenetic comparison of $F$-Box $(F B X)$ gene superfamily within the plant kingdom reveals divergent evolutionary histories indicative of genomic drift. PLOS ONE 6:e16219. doi: 10.1371/journal.pone.0016219

Kong, H., Landherr, L. L., Frohlich, M. W., Leebens-Mack, J., Ma, H., and Depamphilis, C. W. (2007). Patterns of gene duplication in the plant SKP1 gene family in angiosperms: evidence for multiple mechanisms of rapid gene birth. Plant J. 50, 873-885. doi: 10.1111/j.1365-313X.2007. 03097.x

Lechner, E., Xie, D., Grava, S., Pigaglio, E., Planchais, S., Murray, J. A., et al (2002). The AtRbxl protein is part of plant SCF complexes, and its downregulation causes severe growth and developmental defects. J. Biol. Chem. 277, 50069-50080. doi: 10.1074/jbc.M204254200

Lee, J. H., Terzaghi, W., Gusmaroli, G., Charron, J. B., Yoon, H. J., Chen, H., et al. (2008). Characterization of Arabidopsis and rice DWD proteins and their roles as substrate receptors for CUL4-RING E3 ubiquitin ligases. Plant Cell 20, 152-167. doi: 10.1105/tpc.107.055418

Lima, M. E. F., Eloy, N. B., Pegoraro, C., Sagit, R., Rojas, C., et al. (2010) Genomic evolution and complexity of the Anaphase-promoting Complex (APC) in land plants. BMC Plant Biol. 10:254. doi: 10.1186/1471-222910-254

Marín, I. (2010). Diversification and specialization of plant RBR ubiquitin ligases. PLoS ONE 5:e11579. doi: 10.1371/journal.pone.0011579

Merlet, J., Burger, J., Gomes, J. E., and Pintard, L. (2009). Regulation of cullinRING E3 ubiquitin-ligases by neddylation and dimerization. Cell Mol. Life Sci. 66, 1924-1938. doi: 10.1007/s00018-009-8712-7

Mizushima, N. (2020). The ATG conjugation systems in autophagy. Curr. Opin. Cell Biol. 63, 1-10. doi: 10.1016/j.ceb.2019.12.001

Saleme, M. L. S., Andrade, I. R., and Eloy, N. B. (2021). The role of AnaphasePromoting Complex/Cyclosome (APC/C) in plant reproduction. Front. Plant Sci. 12:642934. doi: 10.3389/fpls.2021.642934

Schroeder, D. F., Gahrtz, M., Maxwell, B. B., Cook, R. K., Kan, J. M., Alonso, J. M., et al. (2002). De-etiolated 1 and damaged DNA binding protein 1 interact to regulate Arabidopsis photomorphogenesis. Curr. Biol. 12, 1462-1472. doi: 10.1016/S0960-9822(02)01106-5

Stephani, M., Picchianti, L., and Dagdas, Y. (2021). C53 is a cross-kingdom conserved reticulophagy receptor that bridges the gap between selective autophagy and ribosome stalling at the endoplasmic reticulum. Autophagy 17, 586-587. doi: 10.1080/15548627.2020.1846304

Stone, S. L., Hauksdóttir, H., Troy, A., Herschleb, J., Kraft, E., and Callis, J. (2005). Functional analysis of the RING-type ubiquitin ligase family of Arabidopsis. Plant Physiol. 137, 13-30. doi: 10.1104/pp.104.052423

Trujillo, M. (2018). News from the PUB: plant U-box type E3 ubiquitin ligases. J. Exp. Bot. 69, 371-384. doi: 10.1093/jxb/ erx411 
Vierstra, R. D. (2009). The ubiquitin-26S proteasome system at the nexus of plant biology. Nat. Rev. Mol. Cell Biol. 10, 385-397. doi: 10.1038/ nrm2688

Vierstra, R. D. (2012). The expanding universe of ubiquitin and ubiquitin-like modifiers. Plant Physiol. 160, 2-14. doi: 10.1104/pp.112. 200667

Witting, K. F., and Mulder, M. P. C. (2021). Highly specialized ubiquitin-like modifications: shedding light into the UFM1 enigma. Biomolecules 11:255. doi: 10.3390/biom11020255

Conflict of Interest: The authors declare that the research was conducted in the absence of any commercial or financial relationships that could be construed as a potential conflict of interest.
Publisher's Note: All claims expressed in this article are solely those of the authors and do not necessarily represent those of their affiliated organizations, or those of the publisher, the editors and the reviewers. Any product that may be evaluated in this article, or claim that may be made by its manufacturer, is not guaranteed or endorsed by the publisher.

Copyright $\odot 2021$ Gingerich, Hellmann, Christians and Stone. This is an open-access article distributed under the terms of the Creative Commons Attribution License (CC $B Y)$. The use, distribution or reproduction in other forums is permitted, provided the original author(s) and the copyright owner(s) are credited and that the original publication in this journal is cited, in accordance with accepted academic practice. No use, distribution or reproduction is permitted which does not comply with these terms. 